\title{
AKT plays a pivotal role in the acquisition of resistance to 5-fluorouracil in human squamous carcinoma cells
}

\author{
FUKKA YOU $^{1}$, KENTA AOKI ${ }^{2}$, YATSUJI ITO ${ }^{2}$ and SHIGERU NAKASHIMA ${ }^{1}$ \\ Departments of ${ }^{1}$ Cell Signaling, Division of Cell and Molecular Biology, and ${ }^{2}$ Otorhinolaryngology, \\ Gifu University Graduate School of Medicine, Yanagido 1-1, Gifu 501-1194, Japan
}

Received October 16, 2008; Accepted February 13, 2009

DOI: 10.3892/mmr_00000145

\begin{abstract}
Fluorouracil (5-FU) is a widely used chemotherapeutic agent that inhibits the growth and initiates the apoptosis of epithelial tumors, including squamous cell carcinoma of the head and neck region. However, resistance to this drug is often observed in a clinical setting. The primary mode of action of 5-FU is believed to be the inhibition of thymidylate synthase. Overexpression of the enzymes involved in thymidine synthesis has been shown in some cases to be associated with resistance. However, the detailed mechanisms of resistance of squamous cell carcinoma are not fully understood. In the present study, we examined the involvement of survival signaling pathways in the resistance of squamous carcinoma cells to 5-FU. 5-FU induced the activation of the ERK and Akt kinases in UM-SCC-23 human squamous carcinoma cells, indicating that this anticancer drug activates survival signaling pathways as well as apoptotic signals. In 5-FU-resistant UM-SCC-23 cells established by our group, ERK and Akt signals were constitutively activated. U0126 is an inhibitor of MEK, which is an upstream activator for ERK. U0126 failed to sensitize resistant UM-SCC-23 cells to 5-FU-induced apoptotic cell death. This is in sharp contrast to LY294002, which is an inhibitor of phosphatidylinositol 3-kinase, an upstream activator for Akt. LY294002 drastically enhanced 5-FU-induced apoptotic cell death in resistant UM-SCC-23 cells. These results indicate that the Akt survival signal plays an important role in the resistance of squamous carcinoma cells to 5-FU treatment, and suggest that the modification of Akt activity might provide a new strategy for human 5-FU-resistant squamous carcinoma therapy.
\end{abstract}

\section{Introduction}

Squamous cell carcinoma (SCC) is the largest pathogenic subpopulation among malignant tumors of the head and neck

Correspondence to: Dr Fukka You, Department of Cell Signaling, Division of Cell and Molecular Biology, Gifu University Graduate School of Medicine, Yanagido 1-1, Gifu 501-1194, Japan

E-mail: fy6171@cc.gifu-u.ac.jp

Key words: 5-fluorouracil, drug resistance, squamous cell carcinoma, Akt, ERK region. 5-Fluorouracil (5-FU) is a widely used chemotherapeutic agent that inhibits the growth and initiates the apoptosis of epithelial tumors, including colorectal, breast, ovarian and head and neck cancer. 5-FU-based chemotherapy improves the overall and disease-free survival of patients with these types of cancer. The primary mode of action of 5-FU is believed to be thymidylate synthase inhibition, which results in a reduction in the thymidine pool and an increase in the uracil pool, leading in turn to the inhibition of DNA synthesis (1). Treatment of tumors with DNA-damaging agents such as 5-FU is initially an effective means of arresting malignancy. However, following the initial success of 5-FU in preventing tumor progression, resistance to further treatment with the drug often occurs. Several mechanisms have been proposed to explain 5-FU resistance, including the overexpression of thymidylate synthase (1), increased drug efflux from cancer cells by ATP-dependent transporters (2), and the activities of Bcl-XL (3) or MCL1 (4), anti-apoptotic members of the Bcl-2 family. However, these mechanisms cannot fully account for 5-FU resistance. Thus, the identification of new molecular targets involved in 5-FU resistance will allow the development of novel approaches to the treatment of cancer.

Recent studies have indicated that additional pathways activating anti-apoptotic and/or survival signals might protect tumors from apoptosis induced by chemotherapeutic agents. In particular, the survival signals initiated by phosphatidylinositol 3-kinase (PI3K) (5) and Raf/ERK (6) have been characterized. The serine/threonine kinase Akt is a major effector molecule, which is activated following the activation of PI3K (7) by phosphatidylinositol-dependent kinases after its recruitment to the cellular membrane by the lipid products produced by PI3K. Activated Akt can phosphorylate many downstream targets, including BAD, caspase-9, ribosomal p70 S6 kinase (p70S6K) and forkhead transcription factor. The activation of Raf is also initiated by translocation to the cellular membrane, where it may be phosphorylated by certain kinases (8). Downstream of Raf is MEK1, a dual serine/threonine and tyrosine kinase. MEK further transmits the signal to the extracellular signalregulated kinases ERK1 and ERK2. Activated ERK1/ERK2 can phosphorylate the p90 ribosomal S6 kinase and other CREB kinases. Therefore, the PI3K/Akt and Raf/MEK/ERK signal transduction cascades can transduce signals from the membrane to the cell interior, in some cases to DNA transcription factors. Consequently, these two signaling pathways block apoptosis, either independently or synergistically. 
In this study, we i) examined whether 5-FU activates the survival signaling pathways Raf/ERK and PI3K/Akt, and ii) observed the changes these pathways undergo in 5-FUresistant SCC. The results obtained indicate that 5-FU activates Ras and its downstream effectors Raf/MEK/ERK and Akt in SCC. In 5-FU-resistant SCC, the Ras/Raf/MEK/ERK and Akt pathways were constitutively activated. U0126, a MEK inhibitor, had minimal effect on 5-FU-induced cell death in both parent and 5-FU-resistant SCC. In contrast, 5-FUinduced death was drastically enhanced by the blockade of the Akt signaling pathway by LY294002. These results suggest that the survival signal, which is conducted by Akt, plays an important role in the resistance of SCC to 5-FU treatment.

\section{Materials and methods}

Materials. The UM-SCC-23 (SCC23) human head and neck squamous cell carcinoma cell line was a kind gift from Dr Thomas E. Carey (Laboratory of Head and Neck Cancer Biology, University of Michigan). 5-FU was supplied by Sanofi Aventis (Tokyo, Japan). 3-(4,5-Dimethylthiazol2-yl)-2,5-diphenyltetrazolium bromide and Dulbecco's modified Eagle's medium (DMEM) were obtained from Sigma (St. Louis, MO, USA). U0126 was from Wako (Osaka, Japan) and LY294002 from Promega (Madison, WI, USA). Antibodies against Akt, phosphorylated-Akt, Bad, phosphorylated-Bad and MEK1/2, and the Phospho-ERK1/2 Pathway Sampler Kit, were purchased from Cell Signaling Technology (Beverly, MA, USA). The c-Raf antibody was from Pharmingen (San Diego, CA, USA), and the c-H-Ras antibody from Oncogene Research Products (Cambridge, MA, USA). Antibodies against poly(ADP-ribose)polymerase (PARP), p70S6K and phosphorylated-p70S6K were obtained from Santa Cruz Biotechnology (Santa Cruz, CA, USA). Immobilon-P membrane filters were from Millipore (Bedford, MA, USA). ECL Western blot detection reagents were from NEN (Boston, MA, USA). Fetal bovine serum (FBS) and penicillin/streptomycin were from ICN (Aurora, OH, USA). The EZ-Detect ${ }^{\text {TM }}$ Ras Activation Kit was purchased from Pierce (Rockford, IL, USA).

Cell culture. SCC23 cells were maintained in DMEM supplemented with $10 \%$ FBS, $100 \mathrm{U} / \mathrm{ml}$ penicillin and $100 \mu \mathrm{g} / \mathrm{ml}$ streptomycin (FBS/DMEM) in a humidified atmosphere containing $5 \% \mathrm{CO}_{2}$ at $37^{\circ} \mathrm{C}$. For the experiments, cells $\left(10 \times 10^{5}\right)$ were placed in $100-\mathrm{mm}$ dishes and cultured for $24 \mathrm{~h}$ in FBS/ DMEM, then for another $24 \mathrm{~h}$ in serum-free DMEM (9). The cells were then treated with 5-FU. When necessary, inhibitors were added to the culture medium $1 \mathrm{~h}$ prior to the addition of the anticancer drug.

Cells resistant to 5-fluorouracil. SCC23 cells $\left(10 \times 10^{5}\right.$ in 100-mm dishes) cultured for $24 \mathrm{~h}$ in FBS-DMEM were first treated with $1 \mu \mathrm{g} / \mathrm{ml} \mathrm{5-FU}$ for $24 \mathrm{~h}$. After the medium was removed, the cells were washed twice with phosphate-buffered saline (PBS) and were then incubated in FBS/DMEM for 5 days. This procedure was repeated 5 times until the multiplication abilities of the cells were not affected by the anticancer drug. 5-FU concentrations were increased stepwise from 1.0 to $2.5 \mu \mathrm{g} / \mathrm{ml}$, then to a final $5.0 \mu \mathrm{g} / \mathrm{ml}$. After a total of 20 weeks, the surviving cells were designated as 5-FUresistant SCC cells (reSCC23).

Western blot analysis. Total cellular protein extracts were used for Western blot analysis $(9,10)$. For the preparation of cell extracts, cells were sonicated in RIPA buffer $(25 \mathrm{mM}$ HEPES pH 7.4, 1\% Triton-X, $150 \mathrm{mM} \mathrm{NaCl}, 0.1 \%$ sodium dodecylsulfate, $0.5 \%$ sodium deoxychlolate, $10 \mathrm{mM}$ EDTA, $1 \mathrm{mM}$ phenylmethylsulfonyl fluoride and $10 \mu \mathrm{g} / \mathrm{ml}$ leupeptin) containing phosphatase inhibitors: $1 \mathrm{mM}$ sodium orthovanadate, $10 \mathrm{mM}$ sodium fluoride and $0.1 \mathrm{mM}$ sodium molybdate. Extracted proteins were separated by sodium dodecylsulfate polyacrylamide gel electrophoresis and were electrophoretically transferred onto Immobilon-P membranes. The membranes were probed with the indicated primary antibody and then incubated with an anti-rabbit, -goat, or -mouse IgG horseradish peroxidase-coupled secondary antibody. Detection was performed with the ECL system. Band density was quantified by a densitometer (Densitograph, Atto, Tokyo, Japan).

Microculture tetrazolium (MTT) assay. Cell viability was determined by the MTT assay. Cells $\left(1.0 \times 10^{3}\right)$ were plated in 96-well microplates in $100 \mu \mathrm{l}$ FBS/DMEM. Subsequently, $5 \mu \mathrm{g} / \mathrm{ml}$ of 3-(4,5-Dimethylthiazol-2-yl)-2,5-diphenyltetrazolium bromide $(50 \mu \mathrm{l})$ in PBS was added to each well, and the MTT formazan product was solubilized in $100 \mu \mathrm{l}$ of dimethyl sulfoxide. The absorbance of the sample at $560 \mathrm{~nm}$ was measured using a microplate reader (Immuno Mini NJ2300, System Instruments, Tokyo, Japan). The results of the absorbance of the test wells were expressed as fold increase based on the control wells.

Ras activation assay. Ras activation was measured using the EZ-Detect $^{\text {TM }}$ Ras Activation Kit (9). Cells (10x105) were cultured in 100-mm plates, quickly chilled and lysed in lysis/ binding/wash buffer. The cell lysates were incubated with glutathione S-transferase (GST)-fusion protein containing Ras-binding domain (RBD) of Raf-1 (GST-Raf1-RBD) in the presence of $0.1 \mathrm{mM} \mathrm{GTP} \gamma \mathrm{S}$ and $6 \mathrm{mM} \mathrm{MgCl}_{2}$. Activated Ras coupled with GST-Raf1-RBD was recovered with immobilized glutathione disc. Pulled-down active Ras was detected by Western blot analysis using anti-Ras antibody.

\section{Results}

5-Fluorouracil-resistant SCC23 cells in culture. 5-FUresistant SCC cells were established in culture. The SCC23 cells were first treated with $1.0 \mu \mathrm{g} / \mathrm{ml} 5-\mathrm{FU}$ at concentrations that were increased stepwise from 1.0 to $2.5 \mu \mathrm{g} / \mathrm{ml}$, to a final $5.0 \mu \mathrm{g} / \mathrm{ml}$. After a total of 20 weeks, the surviving cells were designated as 5-FU-resistant cells (reSCC23). reSCC23 cells were relatively resistant to the drug as assessed by MTT compared with the parent SCC23 cells when both cells were treated with $2.5 \mu \mathrm{g} / \mathrm{ml} 5$-FU (Fig. 1A). After a 72-h incubation with $2.5 \mu \mathrm{g} / \mathrm{ml} 5-\mathrm{FU},>75 \%$ of the parent SCC23 cells had disappeared. In contrast, $>75 \%$ of reSCC23 cells had survived. Following 5-FU treatment for $48 \mathrm{~h}$, fragmentation and condensation of the nuclei were clearly observed under fluorescent microscopy when the SCC23 cells were stained with Hoechst 
A

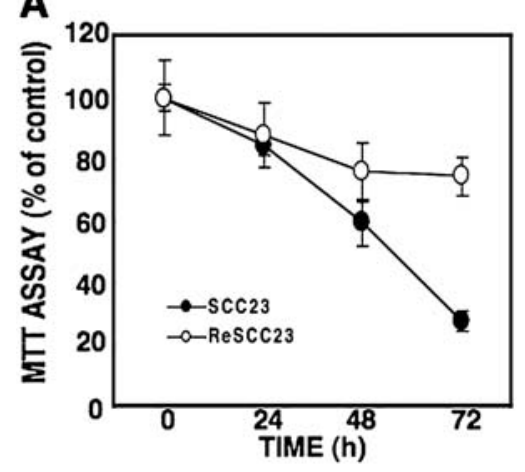

B

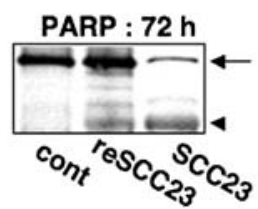

Figure 1. Cytotoxicity of 5-fluorouracil in SCC23 and reSCC23 cells. Cells were treated with $2.5 \mu \mathrm{g} / \mathrm{ml} 5$-FU for the indicated periods of time. (A) Cell viability was assessed by the MTT assay. The results are expressed as the percentage of the untreated controls. Data are the means \pm SD of three independent experiments, each performed in triplicate. (B) PARP degradation. Cells were treated with $2.5 \mu \mathrm{g} / \mathrm{ml} \mathrm{5-FU}$ for $72 \mathrm{~h}$. The extracted proteins were subjected to Western blot analysis with anti-PARP antibody. The arrow indicates PARP and the arrowhead, degraded PARP. The results shown are representative of at least three experiments.

33258 (data not shown). A decrease in PARP ( 115 kDa), a substrate of apoptotic executor caspase-3, coincided with an increase in its cleaved product of $\sim 90 \mathrm{kDa}$ (Fig. 1B), indicating the activation of caspase-3. These results suggest that the 5 -FU-induced decrease in the number of SCC23 cells was due to apoptotic cell death. However, PARP cleavage was minimally observed in reSCC23 cells. Therefore, reSCC23 cells acquired the ability to resist 5-FU-induced apoptotic cell death.

\section{5-Fluorouracil-induced activation of the Raf/MEK/ERK and} Akt signaling pathways in SCC23 cells. Recent studies have indicated that anti-apoptotic and/or survival signals are implicated in the resistence of tumor cells to apoptosis induced by chemotherapeutic agents. Overexpression of an antiapoptotic member of the Bcl-2 family, Bcl-XL, was reported in 5-FU-resistant human colon cancer cells (3). However, the levels of Bcl-2 and Bcl-XL in reSCC23 cells were almost identical to those in parent SCC23 cells (data not shown). Therefore, the Raf/MEK/ERK and Akt signaling pathways were further investigated. Western blot analysis revealed that 5-FU induced the activation of Raf-1, MEK and ERK within $5 \mathrm{~min}$, based on their phosphorylated active forms in response to $2.5 \mu \mathrm{g} / \mathrm{ml} \mathrm{5-FU} \mathrm{in} \mathrm{SCC23} \mathrm{cells} \mathrm{(Fig.} \mathrm{2).} \mathrm{This} \mathrm{suggests} \mathrm{the}$ activation of the Raf/MEK/ERK cascade. Moreover, the activation of Ras, their upstream activator, was also elicited by 5-FU. The drug stimulated another survival signaling pathway that was mediated by protein kinase Akt, since its phosphorylated form and the phosphorylated form of p70S6K, a wellknown substrate of Akt, were also increased in SCC23 cells. Activation of the ERK and Akt pathways lasted for at least $48 \mathrm{~h}$ following 5-FU treatment (Fig. 3). Phosphorylation of a
A

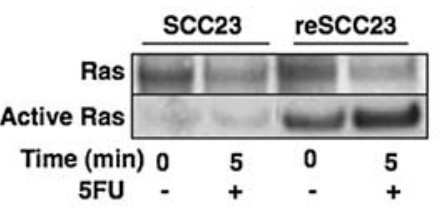

B

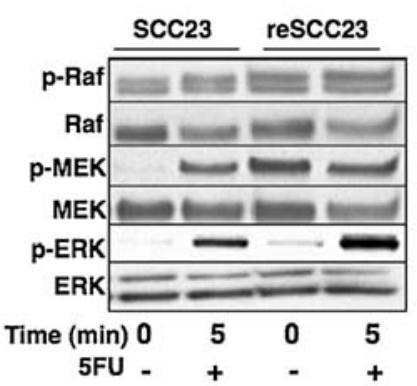

C

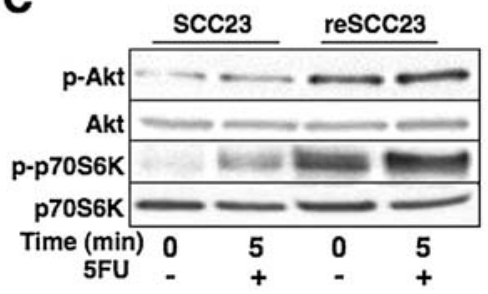

Figure 2. Immediate activation of the Ras/ERK and Akt signaling pathways in response to 5-fluorouracil. SCC23 and reSCC23 cells were treated with $2.5 \mu \mathrm{g} / \mathrm{ml} \mathrm{5-FU}$ for $5 \mathrm{~min}$. (A) Ras activation. Upper panel, Ras protein levels in total cellular extracts; lower panel, amount of activated Ras. The results shown are representative of at least two independent experiments. (B) Activation of the Raf/MEK/ERK pathway. (C) Activation of the Akt pathway. The extracted proteins were subjected to Western blot analysis with the indicated antibodies. The results shown are representative of at least three experiments.

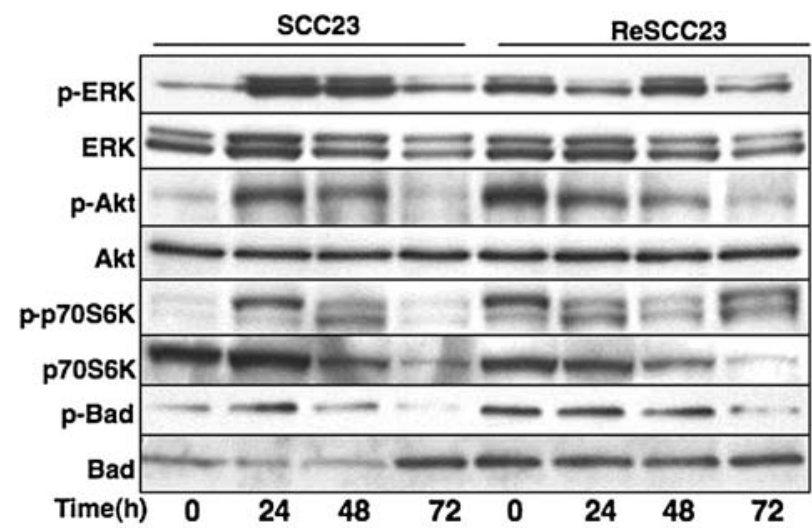

Figure 3. Long-term activation of the Ras/ERK and Akt signaling pathways in response to 5-fluorouracil. SCC23 and reSCC23 cells were treated with $2.5 \mu \mathrm{g} / \mathrm{ml} \mathrm{5-FU}$ for $24-72 \mathrm{~h}$. The extracted proteins were subjected to Western blot analysis with the indicated antibodies. The results shown are representative of at least three experiments.

pro-apoptotic member of the Bcl-2 family, Bad, was stimulated by 5 -FU. Phosphorylation of this pro-apoptotic protein by Akt has been shown to inhibit 5-FU function (11). These results imply that 5-FU activates survival signals as well as apoptotic death pathways in SCC23 cells. 


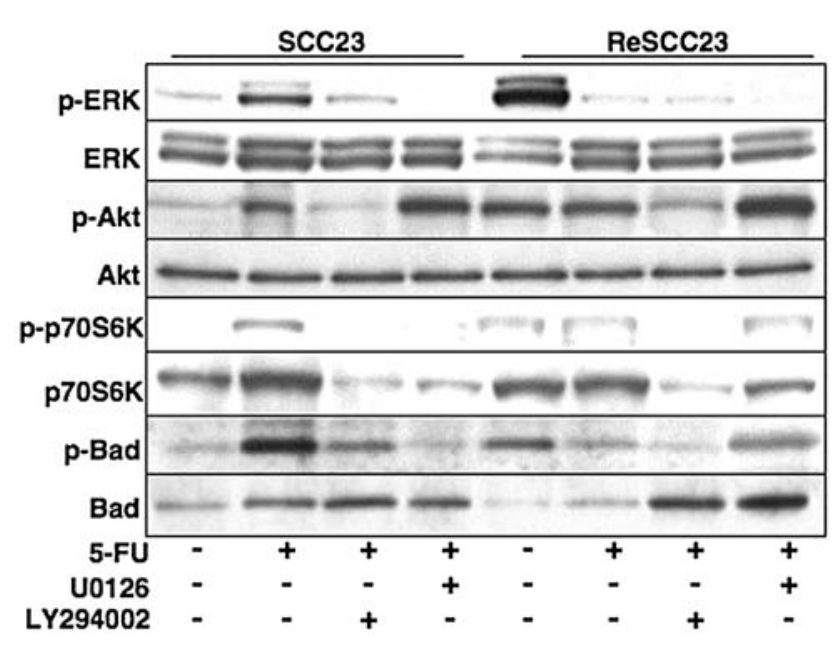

Figure 4. Effects of the PI3K inhibitor LY294002 and MEK1/2 inhibitor U0126 on the 5-fluorouracil-induced activation of the ERK and Akt pathways. SCC23 and reSCC23 cells were pretreated with $10 \mu \mathrm{M} \mathrm{LY} 294002$ or $10 \mu \mathrm{M}$ $\mathrm{U} 0126$ for $1 \mathrm{~h}$ and then exposed to $2.5 \mu \mathrm{g} / \mathrm{ml} 5$-FU for $24 \mathrm{~h}$. The results shown are representative of at least three experiments.

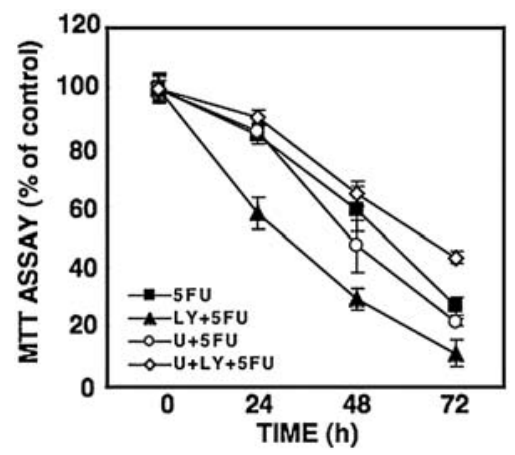

Figure 5. Effects of the PI3K inhibitor LY294002 and MEK1/2 inhibitor U0126 on the 5-fluorouracil-induced death of SCC23 cells. SCC23 cells were pretreated with $10 \mu \mathrm{M} \mathrm{LY} 294002,10 \mu \mathrm{M} \mathrm{U} 0126$ or a combination of the two for $1 \mathrm{~h}$, and then exposed to $2.5 \mu \mathrm{g} / \mathrm{ml} 5$-FU for the indicated periods of time. Cell viability was assessed by the MTT assay. Data are the means \pm SD of three independent experiments, each performed in triplicate.

Constitutive activation of the Raf/MEK/ERK and PI3K/Akt signals in reSCC23 cells. Constitutive activation of ERK and Akt was evident in reSCC23 cells even in the absence of 5-FU treatment (time 0 in Figs. 2 and 3), although the protein levels of these kinases were almost the same as those in the parent SCC23 cells. In addition, Ras was constitutively activated in reSCC23 cells. The protein levels of Ras, Raf and MEK, upstream activators of ERK, were almost the same as those in the parent SCC23 cells. Moreover, phosphorylation of Bad was clearly observed without 5-FU treatment, implying the inactivation of this pro-apoptotic protein in reSCC23 cells.

Sensitization of squamous cell carcinoma to 5-fluorouracilinduced cell death by LY294002, an inhibitor of PI3K. The above results clearly indicate that the growth/survival signaling pathways Raf/MEK/ERK and PI3K/Akt are constitutively activated in reSCC23 cells. We therefore tested for the requirement of these signals for 5-FU resistance using the inhibitors U0126 for MEK and LY294002 for PI3K. U0126 (10 $\mu \mathrm{M})$, a

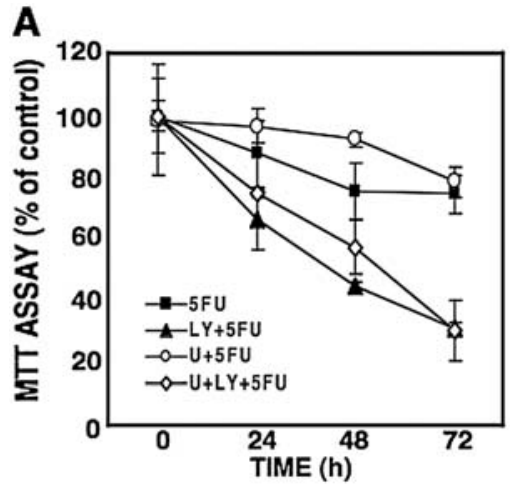

B

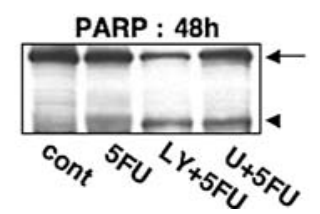

Figure 6. Effects of the PI3K inhibitor LY294002 and MEK1/2 inhibitor U0126 on the 5-fluorouracil-induced cell death of reSCC-23 cells. reSCC23 cells were pretreated with $10 \mu \mathrm{M}$ LY294002, $10 \mu \mathrm{M}$ U0126 or a combination of the two for $1 \mathrm{~h}$, and then exposed to $2.5 \mu \mathrm{g} / \mathrm{ml} \mathrm{5-FU}$ for the indicated periods. (A) Cell viability was assessed by the MTT assay. Data are the means \pm SD of three independent experiments, each performed in triplicate. (B) PARP degradation. The arrow indicates PARP and the arrowhead, degraded PARP. The results shown are representative of at least three experiments.

MEK inhibitor $(9,12)$, nearly abolished the 5-FU-induced activation of ERK in parent SCC23 cells and the constitutive activation of ERK in reSCC23 cells (Fig. 4), although it had little effect on the constitutive phosphorylation of Raf and MEK (data not shown). Notably, this inhibitor rather enhanced the 5-FU-induced phosphorylation of Akt in SCC23 cells and the con-stitutive phosphorylation of Akt in reSCC23 cells. In contrast, $10 \mu \mathrm{M}$ of LY294002, a PI3K inhibitor $(9,12)$, blocked the 5-FU-induced phosphorylation of Akt in SCC23 cells. This inhibitor also suppressed, to a small extent if at all, the 5-FU-induced activation of ERK. In reSCC23 cells, LY294002 suppressed the constitutive phosphorylation of Akt.

As shown in Fig. 5, $10 \mu \mathrm{M}$ U0126, at a concentration that almost abolished ERK activation, did not affect the 5-FUinduced death of parent SCC23 cells. However, LY294002 apparently stimulated 5-FU-induced cell death. In reSCC23 cells, U0126 was not able to stimulate 5-FU-induced cell death (Fig. 6). In sharp contrast, LY294002 markedly enhanced 5-FU-induced cell death in reSCC23 cells. The sensitization effect obtained using a combination of U0126 and LY294002 was almost identical to that obtained with LY294002 alone.

\section{Discussion}

5-FU is used in the treatment of a variety of epithelial tumors, including those of colorectal, breast, ovarian, and head and neck cancer. This anticancer drug initiates apoptosis by targeting thymidylate synthase and by the direct incorporation of 5-FU metabolites into DNA and RNA (1). Typical patient response rates for treatment with this single anticancer agent are between 10 and $30 \%$. The combination of 5-FU with other anticancer drugs has improved response rates. However, chemoresistance is the major obstacle to the success of 5-FU- 
based chemotherapy. Several mechanisms are proposed to be responsible for resistance to 5-FU. Overexpression of thymidylate synthase is thought to be a major resistance-inducing factor (1). The ATP-dependent multidrug resistance protein-5 confers resistance to 5-FU by eluting 5-FU metabolites from cells (2). Overexpression of an anti-apoptotic member of the Bcl-2 family, Bcl-XL, was observed in 5-FU-resistant human colon cancer cells (3). Anther member of the Bcl-2 family, MCL-1, was also shown to protect U2OS cells from 5-FUinduced cytotoxicity (4). More recently, Smug1 DNA glycosylase (13) and MDMX, MDM2-like p53-binding protein (14), has been implicated in 5-FU resistance. Several recent studies $(15,16)$ focused on the identification of molecules which are responsible for the sensitivity and/or resistance to 5-FU by DNA microarrays in order to predict 5-FU sensitivity of cancer cells. These studies will provide useful information for the treatment of cancer patients. At present, however, the precise molecular mechanisms of 5-FU-resistance, particularly in head and neck squamous carcinoma cells, are unknown.

In the present study, we examined the involvement of anti-apoptotic members of the Bcl-2 family, ERK and Akt (molecules which participate in survival signaling), in 5-FUresistance in head and neck squamous carcinoma cells. Overexpression of anti-apoptotic members of the Bcl-2 family has been reported to protect cancer cells from 5-FUinduced cytotoxicity $(3,4)$. We previously showed that Bcl-2 protects cells from apoptosis induced by hypoxia (17) or the anticancer drug etoposide (18). However, we were unable to detect overexpression of Bcl-2 and Bcl-XL in 5-FU-resistant squamous carcinoma (reSCC23) cells established by our group. The Ras/Raf/MEK/ERK and PI3K/Akt pathways are kinase cascades known to promote cellular survival $(6,7,11,12)$. In a previous study, we demonstrated the constitutive activation and involvement of these survival signal cascades in cisplatin resistance in head and neck squamous carcinoma cells (9). Both pathways are also involved in chemoresistance in breast cancer cells (19). In non-small cell lung cancer cells, Akt is constitutively active and promotes cellular survival and resistance to chemotherapy and radiation (20).

In the 5-FU-resistant UM-SCC-23 (reSCC23) cells we established, ERK and Akt signals were constitutively activated, as observed in cisplatin-resistant cells (9). LY294002, an inhibitor for PI3K commonly used to block the Akt signal (12), drastically enhanced 5-FU-induced apoptotic cell death in reSCC23 cells. These results clearly indicate that the Akt survival signal plays an important role in the resistance of squamous carcinoma cells to 5-FU treatment. U0126, an inhibitor for MEK, successfully sensitized cisplatin-resistant cells to cisplatin-induced cell death (9). However, at the same concentrations as those used in cisplatin-resistant cells (9), this inhibitor failed to sensitize reSCC23 cells to 5-FU-induced apoptotic cell death, despite the complete inactivation of ERK. These results suggest that the Raf/MEK/ERK pathway plays a minimal role, if any, in the resistance of squamous carcinoma cells to 5-FU treatment.

In summary, the growth/survival signaling pathways Raf/ MEK/ERK and PI3K/Akt are activated in 5-FU-resistant squamous carcinoma cells. We identified Akt, but not Raf/ MEK/ERK, as a constitutively active survival signal which promotes the resistance of squamous carcinoma cells to 5-FU treatment. Modification of Akt activity may therefore provide a new strategy for human 5-FU-resistant squamous carcinoma therapy.

\section{References}

1. Longley DB, Harkin DP and Johnston PG: 5-Fluorouracil: mechanism of action and clinical strategies. Nat Rev Cancer 3: 330-338, 2003.

2. Pratt S, Shepard RL, Kandasamy RA, Johnston PA, Perry W III and Dantzig AH: The multidrug resistance protein 5 (ABCC5) confers resistance to 5-fluorouracil and transports its monophosphorylated metabolites. Mol Cancer Ther 4: 855-863, 2005.

3. Zhu H, Guo W, Zhang L, Davis JJ, Teraishi F, Wu S, Cao X, Daniel J, Smythe WR and Fang B: Bcl-XL small interfering RNA suppresses the proliferation of 5-fluorouracil-resistant human colon cancer cells. Mol Cancer Ther 4: 451-456, 2005.

4. Graidist P, Phongdara A and Fujise K: Antiapoptotic protein partners fortilin and MCL1 independently protect cells from 5-fluorouracil-induced cytotoxicity. J Biol Chem 279: 40868-40875, 2004.

5. Datta SR, Brunet A and Greenberg ME: Cellular survival: a play in three Akts. Genes Dev 13: 2905-2927, 1999.

6. Erhardt P, Schremser EJ and Cooper GM: B-Raf inhibits programmed cell death downstream of cytochrome c release from mitochondria by activating the MEK/ERK pathway. Mol Cell Biol 19: 5308-5315, 1999.

7. Khwaja A, Rodrigues-Vicana P, Wennstrom S, Warne PH and Downward J: Matrix adhesion and Ras transformation both activate a phosphoinositide 3-OH kinase and protein kinase B/Akt cellular survival pathway. EMBO J 16: 2783-2793, 1997.

8. Marais R, Light Y, Paterson HF and Marshall CJ: Ras recruits Raf-1 to the plasma membrane for activation by tyrosine phosphorylation. EMBO J 14: 3136-3145, 1995.

9. Aoki K, Ogawa T, Ito Y and Nakashima S: Cisplatin activates survival signals in UM-SCC-23 squamous cell carcinoma and these signal pathways are amplified in cisplatin-resistant squamous cell carcinoma. Oncol Rep 11: 375-379, 2004.

10. You F, Osawa Y, Hayashi S and Nakashima S: Immediate early gene IEX-1 induces astrocytic differentiation of U87-MG human glioma cells. J Cell Biochem 100: 256-265, 2007.

11. Del Peso L, Gonzalez-Garcia M, Page C, Herrera R and Nunez G: Interleukin-3-induced phosphorylation of BAD through the protein kinase Akt. Science 278: 687-689, 1997.

12. Shelton JG, Steelman LS, Lee JT, Knapp SL, Blalock WL, Moye PW, Franklin RA, Pohnert SC, Mirza AM, McMahon M and McCubrey JA: Effect of RAF/MEK/ERK and PI3K/AKT signal transduction pathways on the abrogation of cytokinedependence and prevention of apoptosis in hematopoietic cells. Oncogene 22: 2478-2492, 2003.

13. An Q, Robins P, Lindahl T and Barnes DE: 5-Fluorouracil incorporated into DNA is excised by the Smug1 DNA glycosylase to reduce drug cytotoxicity. Cancer Res 67: 940-945, 2007.

14. Gilkes DM, Chen L and Chen J: MDMX regulation of p53 response to ribosomal stress. EMBO J 25: 5614-5625, 2006.

15. Wang W, Cassidy J, O'Brien V, Ryan KM and Collie-Duguid E: Mechanistic and predictive profiling of 5-fluorouracil resistance in human cancer cells. Cancer Res 64: 8167-8176, 2004.

16. De Angelis PM, Svendsrud DH, Kravik KL and Stokke T: Cellular response to 5-fluorouracil (5-FU) in 5-FU-resistant colon cancer cell lines during treatment and recovery. Mol Cancer 5: 20, 2006.

17. Yoshimura S, Banno Y, Nakashima S, Takenaka K, Sakai H, Nishimura Y, Sakai N, Shimizu S, Eguchi Y, Tsujimoto Y and Nozawa Y: Ceramide formation leads to caspase-3 activation during hypoxic PC12 cell death: Inhibitory effects of Bcl-2 on ceramide formation and caspase-3 activation. J Biol Chem 268: 6921-6927, 1998

18. Sawada M, Nakashima S, Banno Y, Yamakawa H, Takenaka K, Shinoda J, Nishimura Y, Sakai N and Nozawa Y: Influence of Bax or Bcl-2 overexpression on the ceramide-dependent apoptotic pathway in glioma cells. Oncogene 19: 3508-3520, 2000.

19. Jin W, Wu L, Liang K, Liu B, Lu Y and Fan Z: Roles of the PI-3K and MEK pathways in Ras-mediated chemoresistance in breast cancer cells. Br J Cancer 89: 185-191, 2003.

20. Brognard J, Clark AS, Ni Y and Dennis PA: Akt/protein kinase $\mathrm{B}$ is constitutively active in non-small cell lung cancer cells and promotes cellular survival and resistance to chemotherapy and radiation. Cancer Res 61: 3986-3997, 2001. 